\title{
The Product of Red Blood Cells and Hematocrit Can Be Used as a Novel Indicator of Impaired Fasting Blood Glucose Status
}

This article was published in the following Dove Press journal: Diabetes, Metabolic Syndrome and Obesity: Targets and Therapy

\author{
Ling Feng* \\ Haishan Chen* \\ Jianhui Chen \\ Chongxiang Xiong \\ Xiaofei Shao \\ Xin Wang \\ Jing Ning (D) \\ Zhicong Xiang \\ Xuan Wang \\ Tong Chen \\ Hua Xiao \\ Hongjuan Tang \\ Xiaolin Li \\ Guobao Hong \\ Hequn Zou \\ Department of Nephrology, The Third \\ Affiliated Hospital, Southern Medical \\ University, Guangzhou 510630, People's \\ Republic of China \\ *These authors contributed equally to \\ this work.
}

Correspondence: Hequn Zou;

Xiaofei Shao

Department of Nephrology, The Third Affiliated Hospital, Southern Medical

University, 183, Zhongshan West Avenue,

Tianhe District, Guangzhou 510630,

People's Republic of China

Email Hequnzou@hotmail.com;

4I3I5II87@qq.com
Objective: To explore whether the red blood cell count multiplied by hematocrit index (RBCHct) in blood routine parameters can indicate the risk of impaired fasting blood glucose (IFG), and whether it is related to insulin resistance and inflammation.

Methods: In this cross-sectional study, previous history of diabetes was excluded, and people with normal and impaired IFG were included. We use Spearman analysis to evaluate the correlation between RBCHct index and fasting plasma glucose, insulin resistance homeostasis model assessment (HOMA-IR), and hypersensitive C-reactive protein (hs-CRP). Binary logistic regression analysis was used to evaluate the $\mathrm{RBCHct}$ index for assessing the potential risk of IFG, and the receiver operating characteristic (ROC) curve was used to evaluate the RBCHct index for diagnosing insulin resistance and chronic low-grade inflammatory efficacy among those with IFG.

Results: Correlation analysis showed that the RBCHct index and fasting plasma glucose $(\mathrm{r}=0.088, \mathrm{P}=0.003)$; HOMA-IR $(\mathrm{r}=0.199, \mathrm{P}<0.001)$; and hs-CRP $(\mathrm{r}=0.097, \mathrm{P}=0.001)$ were positively correlated. After adjusting for confounding factors, the risk of IFG in the third and fourth quartiles of the RBCHct index increased to 1.889 and 3.048 times. The area under the ROC curve of the RBCHct index for diagnosis of insulin resistance state (HOMA-IR) was 0.695 ( $\mathrm{p}<0.001)$, and the area under the ROC curve of the RBCHct index for the diagnosis of chronic low-inflammatory state (hs-CRP) was 0.641 ( $\mathrm{P}=0.010)$.

Conclusion: The RBCHct index may be a potential indicator for assessing the risk of prediabetes and is closely related to whether the body is in a state of insulin resistance and inflammation under IFG.

Keywords: diabetes mellitus, erythrocyte, RBCHct index, insulin resistance, inflammation

\section{Introduction}

In a national epidemiological survey conducted in China in 2010, the overall prevalence of diabetes was estimated to be $10.9 \%$, and the prevalence of prediabetes was $35.7 \% .^{1}$ The number of patients with diabetes showed an upward trend, and the pre-diabetes population occupied a large proportion of these patients. The task of preventing and treating the occurrence and progression of diabetes is complex. People with diabetes and pre-diabetes are in a chronic low-inflammatory state, which may be the main reason for the development of diabetes and related complications. Under hyperglycemic state, a series of pathological changes occur in vivo, such as oxidative stress, vascular endothelial damage, and the accumulation of glycosylation end products. ${ }^{2-4}$ In animal experiments, it was confirmed that in 
the pre-diabetes period, high amylin secretion participates in the morphological changes of the erythrocytes, and increased erythropoietin secretion leads to increased production of red blood cells. ${ }^{5}$ Electron microscopy also revealed abnormal erythrocyte morphology in prediabetic samples. ${ }^{6}$ In addition, the oxygen exchange capacity of the microcirculation is closely related to red blood cells and hematocrit. ${ }^{7}$ With the morphological changes and functional decline of red blood cells, the red blood cells cannot pass smoothly through the capillaries and appear to aggregate, resulting in microcirculation ischemia and hypoxia, oxidative stress reaction, and capillary endothelial damage, which can further lead to platelet adhesion and white blood cells aggregation, subsequently producing an inflammatory response. It can be speculated that the red blood cell count and hematocrit at the local microcirculation are involved in the initiation of the diabetes, which may be one of the causes of chronic low-grade inflammation in prediabetic patients. We aimed to evaluate whether changes in the red blood cell count multiplied by the hematocrit index (RBCHct) can indicate the risk of impaired fasting blood glucose (IFG), and is closely related to insulin resistance and chronic low-grade inflammation in the IFG state.

\section{Methods}

\section{Study Population}

The data for this cross-sectional survey were derived from an epidemiological survey conducted in six communities in Wanzhai Town, Zhuhai City, southern China from January to April 2018. Adult residents aged $\geq 18$ years living in these six communities were invited to participate in this survey and 1140 residents met the criteria to participate in the survey. The study was conducted in accordance with the tenets of the Helsinki Declaration and was approved by the Ethics Committee of the Third Affiliated Hospital of Southern Medical University, China. All subjects provided written informed consent. The subjects of the study were people with normal blood glucose and those with IFG. Individuals who were diagnosed with diabetes and showed fasting blood glucose $\geq 7.0 \mathrm{mmol} / \mathrm{L}$, those with hematological system diseases, infectious disease, chronic kidney disease, the estimated glomerular filtration rate (eGFR) $\leq 60 \mathrm{~mL} / \mathrm{min} / 1.73 \mathrm{~m}^{2}$, and/or those on current antiplatelet drugs and uric acid-lowering drugs were excluded.

\section{Data Collection}

All medical staff and medical students participating in this study received standardized training. Basic information regarding age, sex, smoking, drinking, education level, sports, disease history, and drug use was obtained through the questionnaire.

\section{Anthropometry}

Human body measurements were performed according to the recommendations of the World Health Organization. A mercury sphygmomanometer was used to measure the blood pressure of each participant in the left arm, after the patient had rested for at least $5 \mathrm{~min}$; the mean value of three measurements was considered. The participants were requested to wear light clothes and stand and breathe naturally; then, the waist circumference (WC) between the lower costal margin of the midline of the axilla and the iliac crest was measured.

\section{Laboratory Variables}

The included analysis indicators are as follows: (i) Blood routine parameters: ethylenediaminetetraacetic acid (EDTA) anticoagulation test tube was used to collect blood samples for routine blood parameter analysis including red blood cell count (RBC), hemoglobin (HGB), hematocrit (Hct), red blood cell distribution width (RDW-SD), coefficient of variation of erythrocyte distribution width (RDW-CV), white blood cell count (WBC), neutrophil count, and lymphocyte count; (ii) Prediabetes-related indicators: standard enzymatic method was used to measure fasting blood glucose and electrochemiluminescence method was used to measure insulin levels; (iii) Blood lipids: colorimetric method was used to determine triglycerides (TG), total cholesterol (TC), high-density lipoprotein cholesterol (HDL-C), low-density lipoprotein cholesterol (LDL-C); (iv) Biochemical indicators: colorimetric method was used to determine serum total protein and albumin (ALB); (v) Oxidative stress and inflammation risk indicators: ELISA was used to measure superoxide dismutase (SOD) and interleukin 6 (IL-6), and immunoturbidimetry was used to measure hs-CRP concentration.

\section{Definition and Calculation Formula}

We divided the target population into two groups, the normal group ( $\mathrm{n}=992$ cases) had fasting plasma glucose $<5.6 \mathrm{mmol} / \mathrm{L}$ $(100 \mathrm{mg} / \mathrm{dL})$, and the IFG group ( $\mathrm{n}=148$ cases) had fasting plasma glucose $\geq 5.6 \mathrm{mmol} / \mathrm{L}$ and $<7.0 \mathrm{mmol} / \mathrm{L}$ $(100-126 \mathrm{mg} / \mathrm{dL}) .{ }^{8}$ The HOMA-IR value was calculated as 
fasting plasma glucose $(\mathrm{mmol} / \mathrm{L}) \times$ fasting insulin $(\mathrm{mU} / \mathrm{L}) /$ 22.5; the body mass index (BMI) was calculated as weight $(\mathrm{kg}) /$ height in squared meters $\left(\mathrm{m}^{2}\right)$. The modified MDRD equation was used to calculate the eGFR: $175 \mathrm{x}$ (Scr) - 1.234 $\mathrm{x}$ (age) - $0.179 \mathrm{x}$ (if female, $\mathrm{x}$ 0.79). The RBCHct was calculated as (Red blood cell count $\left.\times 10^{12} / \mathrm{L}\right) \times$ hematocrit (Hct). Grouping based on RBCHct interquartile range was as follows: Q1 (RBCHct<1.85), Q2 (1.85 $\leq$ RBCHct<2.06), Q3 $(2.06 \leq \mathrm{RBCHct}<2.3$ ), and $\mathrm{Q} 4$ (RBCHct $\geq 2.3$ ). Grouping based on HOMA-IR index was as follows: normal group $(n=54)$, HOMA-IR $\leq 2.69$ and insulin resistance group $(n=94)$, HOMA-IR $>2.69 .{ }^{9}$ Grouping based on hs-CRP value was as follows: normal group $(\mathrm{n}=115)$, hs-CRP $\leq 3.0 \mathrm{mg} / \mathrm{L}$ and chronic low-grade inflammation group $(n=33)$, hs-CRP $>3.0 \mathrm{mg} / \mathrm{L}^{10,11}$

\section{Statistical Analysis}

The SPSS (version 20) and MedCalc statistical software were used to analyze the data. Continuous variables with normal distribution are displayed as mean $\pm \mathrm{SD}$, and the independent sample $t$-test and ANOVA were used for comparison of differences, followed by Bonferroni correction for pairwise comparison. Continuous variables with nonnormal distribution are presented as median (25th-75th percentile), Mann-Whitney $U$-test and Kruskal-Wallis $\mathrm{H}(\mathrm{K})$ were used for difference comparison; countable data are expressed as percentage, using the chi-square test for difference comparison. Spearman correlation was for correlation analysis of RBCHct index and related indicators. Binary logistic regression was used for RBCHct index assessing prediabetic potential risk analysis to establish three models: Model 1 is not adjusted; Model 2 is adjusted for age, sex, BMI, and WC; and Model 3 is adjusted for age, sex, BMI, waist circumference, smoking, drinking, educational level, physical exercise, TG, and TC. The results are expressed as odds ratio (OR) and $95 \%$ confidence interval (CI). ROC curve analysis was used to determine RBCHct index for diagnosis of insulin resistance and chronic lowgrade inflammation. Two-tailed $\mathrm{p}$-value $<0.05$ was considered to indicate statistical significance.

\section{Results}

Table 1 shows the description of baseline characteristics of the normal glucose and IFG groups. It can be seen from Table 1, compared with the normal group, the IFG group is older and has higher BMI, WC, blood pressure, and HOMA-IR ( $<<0.001)$; TG, TC, WBC, lymphocytes, SOD and ALB all increased $(\mathrm{p}<0.05)$. In addition, in the IFG group, RBCHct, RBC, HGB, and Hct increased $(\mathrm{p}<0.05)$, RDW-SD decreased $(\mathrm{p}=0.049)$, and there was no difference in RDW-CV.

Table 2 provides a description of the correlation between RBCHct index and human body status indicator. Correlation analysis showed that the RBCHct index and fasting blood glucose $\quad(\mathrm{r}=0.088, \quad \mathrm{P}=0.003) ;$ blood insulin $\quad(\mathrm{r}=0.192$, $\mathrm{P}<0.001)$; HOMA-IR ( $\mathrm{r}=0.199, \mathrm{P}<0.001)$; WBC $(\mathrm{r}=0.305$, $\mathrm{P}<0.001)$; lymphocyte count $(\mathrm{r}=0.205, \mathrm{P}<0.001)$; neutrophil count ( $\mathrm{r}=0.254, \mathrm{P}<0.001)$; hs-CRP $(\mathrm{r}=0.097, \mathrm{P}=0.001)$; SOD $(\mathrm{r}=0.292, \mathrm{P}<0.001) ; \mathrm{ALB}(\mathrm{r}=0.230, \mathrm{P}<0.001)$; $\mathrm{SBP}(\mathrm{r}=0.159$, $\mathrm{P}<0.001)$; DBP $(\mathrm{r}=0.244, \mathrm{P}<0.001)$; $\mathrm{WC}(\mathrm{r}=0.311, \mathrm{P}<0.001)$; and BMI $(\mathrm{r}=0.199, \mathrm{P}<0.001)$ were positively correlated. The RBCHct index was negatively correlated with HDL $(\mathrm{r}=-0.241, \mathrm{P}<0.001)$.

Table 3 shows the description of the basic characteristics of the human body with respect to the interquartile range of RBCHct index. For every quartile increase in RBCHct among people, BMI, SBP, DBP, fasting plasma glucose, insulin, HOMA-IR, RBC, Hct, RDW-CV, WBC, neutrophils, lymphocytes, SOD, ALB, triglycerides, cholesterol, the proportion of males, the medical history of smoking and drinking increased $(\mathrm{P}<0.05)$; the level of RDW-SD decreased $(\mathrm{P}<0.05)$; there was no difference in age, exercise, and education level $(\mathrm{P}>0.05)$. The highest quartile of RBCHct was associated with a higher prevalence of IFG $(\mathrm{P}<0.05)$.

The relationship between the interquartile range of RBCHct index and the potential risk of IFG is shown in Table 4 . In the binary logistic regression analysis, IFG is the dependent variable, and the RBCHct quartile is the independent variable. In the unadjusted model 1, compared with the lowest quartile array 1 , the OR at the highest RBCHct quartile was $2.925(\mathrm{P}<0.001)$; in model 2 , after adjusting for age, gender, $\mathrm{BMI}$, and $\mathrm{WC}$, The OR of the highest quartile was $3.427(\mathrm{P}<0.001)$; in Model 3, after adjusting for age, gender, BMI, and WC, smoking, drinking, education (high school or above), physical activity, TG, and TC, the OR of the highest quartile was 3.048 $(\mathrm{P}=0.001)$. RBCHct is independently related to IFG.

The RBCHct index was used to evaluate the efficacy of insulin resistance and chronic low-grade inflammation. The RBCHct index was used as the detection variable, and the occurrence of insulin resistance was used as the outcome variable for ROC curve analysis. The area under the HOMA-IR curve of 95\% CI was 0.695 (0.614-0.768); the optimal cut-off value was 2.002 , sensitivity was $77.66 \%$, specificity was $53.7 \%$, and the Youden index 
Table I Demographic and Clinical Characteristics of the Normal Glucose Group and IFG

\begin{tabular}{|c|c|c|c|}
\hline Variables & $\begin{array}{l}\text { (NG) } \\
(n=992)\end{array}$ & $\begin{array}{l}(I F G) \\
(n=\mid 48)\end{array}$ & $P$ value \\
\hline Age (years) & $54.34 \pm 12.79$ & $59.93 \pm 9.84$ & $P<0.001$ \\
\hline Male, n (\%) & $327(32.96)$ & $51(34.5)$ & 0.709 \\
\hline Current smoker, n (\%) & $117(11.79)$ & $13(8.78)$ & 0.333 \\
\hline $\begin{array}{l}\text { Current alcohol use, } \\
\text { n (\%) }\end{array}$ & $233(23.49)$ & $35(23.65)$ & 0.966 \\
\hline $\begin{array}{l}\text { Education - high school } \\
\text { or above, n (\%) }\end{array}$ & $139(14.01)$ & $14(9.5)$ & 0.155 \\
\hline Physical activity, n (\%) & $532(53.63)$ & $92(62.16)$ & 0.63 \\
\hline BMI, $\mathrm{kg} / \mathrm{m}^{2}$ & $23.9 \pm 3.32$ & $25.23 \pm 3.38$ & $P<0.001$ \\
\hline WC, $\mathrm{cm}$ & $83.65 \pm 9.56$ & $88.44 \pm 9.31$ & $P<0.001$ \\
\hline $\mathrm{SBP}, \mathrm{mmHg}$ & $131.95 \pm 18.91$ & $141.3 \pm 17.55$ & $P<0.001$ \\
\hline DBP, $\mathrm{mmHg}$ & $81.85 \pm 10.34$ & $86.48 \pm 8.94$ & $P<0.001$ \\
\hline $\begin{array}{l}\text { Fasting plasma glucose, } \\
\mathrm{mmol} / \mathrm{L}\end{array}$ & $4.85 \pm 0.37$ & $5.99 \pm 0.34$ & $P<0.00$ I \\
\hline Insulin, $\mathrm{mU} / \mathrm{L}$ & $\begin{array}{l}8.85 \\
(6.38-12.36)\end{array}$ & $\begin{array}{l}12.59 \\
(8.33-16.37)\end{array}$ & $P<0.001$ \\
\hline HOMA-IR & $1.9(1.35-2.72)$ & $\begin{array}{l}3.36 \\
(2.19-4.4)\end{array}$ & $P<0.001$ \\
\hline $\mathrm{RBCHct}$ & $2.09 \pm 0.34$ & $2.19 \pm 0.34$ & 0.001 \\
\hline $\mathrm{RBC}, 10^{12} / \mathrm{L}$ & $4.8 \pm 0.54$ & $4.96 \pm 0.58$ & 0.001 \\
\hline HGB, $g / L$ & $140.05 \pm 13.32$ & $143 \pm 12.52$ & 0.011 \\
\hline Hct (\%) & $0.43 \pm 0.03$ & $0.44 \pm 0.03$ & 0.013 \\
\hline RDW-SD (fl) & $43.1 \pm 3.16$ & $42.55 \pm 2.79$ & 0.049 \\
\hline RDW-CV (\%) & $13.41 \pm 1.42$ & $|3.4| \pm|.4|$ & 0.988 \\
\hline WBC, $10^{9} / \mathrm{L}$ & $6.33 \pm 1.4$ & $6.63 \pm 1.37$ & 0.015 \\
\hline Neutrophil count, $10^{9} / \mathrm{L}$ & $3.35 \pm 1.03$ & $3.5 \pm 1$ & 0.106 \\
\hline Lymphocyte count, $10^{9} / \mathrm{L}$ & $2.28 \pm 0.62$ & $2.44 \pm 0.67$ & 0.005 \\
\hline $\mathrm{IL}-6, \mathrm{pg} / \mathrm{mL}$ & $3.8 \pm 4.14$ & $3.81 \pm 2.05$ & 0.998 \\
\hline hs-CRP, mg/L & $2.32 \pm 4.83$ & $2.49 \pm 3.75$ & 0.682 \\
\hline SOD, U/L & $|52.95 \pm 22.5|$ & $\begin{array}{l}159.99 \\
\pm 35.93\end{array}$ & 0.001 \\
\hline ALB, $g / L$ & $47.9 \pm 2.56$ & $48.57 \pm 2.43$ & 0.003 \\
\hline $\mathrm{TG}, \mathrm{mmol} / \mathrm{L}$ & $\begin{array}{l}1.26 \\
(0.93-1.83)\end{array}$ & $\begin{array}{l}1.56 \\
(1.15-2.36)\end{array}$ & $P<0.001$ \\
\hline $\mathrm{TC}, \mathrm{mmol} / \mathrm{L}$ & $5.46 \pm 1.05$ & $5.7 \pm 1.13$ & 0.012 \\
\hline LDL-C, mmol/L & $3.23 \pm 0.94$ & $3.36 \pm 0.97$ & 0.126 \\
\hline HDL-C, mmol/L & $1.54 \pm 0.34$ & $1.48 \pm 0.36$ & 0.058 \\
\hline
\end{tabular}

Note: Data are presented as mean \pm SD or median (25th-75th percentiles) for continuous variables and as percentage (\%) for countable data and categorical variables.

Abbreviations:: NG, normal blood glucose group; IFG, impaired fasting blood glucose group; BMI, body mass index; WC, waist circumference; SBP, systolic blood pressure; DBP, diastolic blood pressure; HOMA-IR, insulin resistance homeostasis model assessment; RBCHct, red blood cell count multiplied by hematocrit index; RBC, red blood cell count; HGB, hemoglobin; Hct, hematocrit; RDW-SD, red blood cell distribution width; RDW-CV, coefficient of variation of erythrocyte distribution width; WBC, white blood cell count; IL-6, interleukin 6; hs-CRP, hypersensitive C-reactive protein; SOD, superoxide dismutase; ALB, serum albumin; TG, triglycerides; TC, total cholesterol; LDL-C, low-density lipoprotein cholesterol; HDL-C, high-density lipoprotein cholesterol.

was 0.3136 (Figure 1). The RBCHct index was used as the detection variable, and the occurrence of chronic lowgrade inflammation was used as the outcome variable for
Table 2 Description of the Correlation Between RBCHct Index and Human Body Status Indicators

\begin{tabular}{|l|l|l|l|}
\hline Variables & $\mathbf{R}$ & $\mathbf{P}$ value & $\begin{array}{l}\text { Grading of the } \\
\text { Correlation } \\
\text { Results }\end{array}$ \\
\hline Fasting plasma glucose & 0.088 & 0.003 & Small \\
Insulin & 0.192 & $\mathrm{P}<0.00 \mathrm{I}$ & Small \\
HOMA-IR & 0.199 & $\mathrm{P}<0.001$ & Small \\
WBC & 0.305 & $\mathrm{P}<0.001$ & Medium \\
Lymphocyte count & 0.205 & $\mathrm{P}<0.001$ & Small \\
Neutrophil count & 0.254 & $\mathrm{P}<0.001$ & Small \\
Hs-CRP & 0.097 & 0.001 & Small \\
SOD & 0.292 & $\mathrm{P}<0.001$ & Medium \\
ALB & 0.230 & $\mathrm{P}<0.001$ & Small \\
TG & 0.254 & $\mathrm{P}<0.001$ & Small \\
TC & 0.103 & $\mathrm{P}<0.001$ & Small \\
LDL-C & 0.114 & $\mathrm{P}<0.001$ & Small \\
HDL-C & -0.241 & $\mathrm{P}<0.001$ & Small \\
SBP & 0.159 & $\mathrm{P}<0.001$ & Small \\
DBP & 0.244 & $\mathrm{P}<0.001$ & Small \\
WC & 0.311 & $\mathrm{P}<0.001$ & Medium \\
BMI & 0.199 & $\mathrm{P}<0.001$ & Small \\
\hline
\end{tabular}

Abbreviations: HOMA-IR, insulin resistance homeostasis model assessment; WBC, white blood cell count; hs-CRP, hypersensitive C-reactive protein; SOD, superoxide dismutase; ALB, serum albumin; TG, triglycerides; TG, total cholesterol; LDL-C, low-density lipoprotein cholesterol; HDL-C, high-density lipoprotein cholesterol; SBP, systolic blood pressure; DBP, diastolic blood pressure; WC, waist circumference; BMI, body mass index.

ROC curve analysis. The area under the hs-CRP curve of 95\% CI was $0.641(0.558-0.718)$; the optimal cut-off value was 2.1285 , the sensitivity was $50.43 \%$, the specificity was $72.73 \%$, and the Youden index was 0.2316 (Figure 2).

\section{Discussion}

Elevated plasma glucose can change blood routine parameters, the main changes are as follows: (I) Increased red blood cell count. Both erythrocyte membrane protein and hemoglobin can undergo non-enzymatic glycosylation with glucose, and the interaction between them is linearly related, initially forming a reversible glycosylation products precursor (imine), and further oxidation to form an irreversible advanced glycosylation end products. ${ }^{12,13}$ Glycosylated proteins can undergo auto-oxidation to promote the production of superoxide and free radicals. ${ }^{14,15} \mathrm{In}$ this process, red blood cell membrane tubulin subtypes may undergo structural changes; red blood cell membrane $\mathrm{Ca} 2+$ ATPase enzyme, red blood cell membrane $\mathrm{Na}+/ \mathrm{K}+-$ ATPase enzyme, erythrocyte sodium-lithium countertransport (SLC) may have functions obstacle. ${ }^{16,17}$ In addition, 
Table 3 Description of the Basic Characteristics of the Human Body Under the Interquartile Range of RBCHct Index

\begin{tabular}{|c|c|c|c|c|c|}
\hline Variables & Q I $(n=286)$ & Q2 (n=28I) & Q3 (n=286) & Q4 (n=287) & $P$ value \\
\hline Age (years) & $54.69 \pm 13.13$ & $55.87 \pm|2.4|$ & $55.32 \pm 12.69$ & $54.4 \pm 12.1$ & 0.509 \\
\hline Male, n (\%) & $15(5.24)$ & $44(15.66)$ & $107(37.4 I)$ & $212(73.87)$ & $P<0.001$ \\
\hline Current smoker, n (\%) & $10(3.5)$ & $17(6.05)$ & $35(12.24)$ & $68(23.69)$ & $P<0.001$ \\
\hline Current alcohol use, n (\%) & $43(15.03)$ & $43(15.30)$ & $73(25.52)$ & 109(37.98) & $\mathrm{P}<0.00 \mathrm{I}$ \\
\hline Education - high school or above, n (\%) & $42(14.69)$ & $29(10.32)$ & $42(14.69)$ & $40(13.94)$ & 0.365 \\
\hline Physical activity, n (\%) & $153(53.5)$ & $166(59.07)$ & I54 (53.85) & $|5|(52.61)$ & 0.403 \\
\hline IFG (\%) & $21(7.34)$ & $36(|2.8|)$ & $37(12.94)$ & $54(18.82)$ & 0.001 \\
\hline $\mathrm{BMI}, \mathrm{kg} / \mathrm{m}^{2}$ & $23.36 \pm 3.1^{d}$ & $23.95 \pm 3.23^{\mathrm{d}}$ & $23.78 \pm 3.3^{d}$ & $25.2 \pm 3.5^{\mathrm{abc}}$ & $P<0.001$ \\
\hline WC, $\mathrm{cm}$ & $81.27 \pm 8.88^{\mathrm{cd}}$ & $82.5 \pm 9.09^{d}$ & $84.12 \pm 9.27^{\mathrm{ad}}$ & $89.15 \pm 9.5^{\mathrm{abc}}$ & $P<0.001$ \\
\hline SBP, mmHg & $129.39 \pm 19.16^{\mathrm{cd}}$ & $132.02 \pm 19.56^{d}$ & $134.86 \pm 18.89^{a}$ & $136.33 \pm 17.68^{\mathrm{ab}}$ & $P<0.001$ \\
\hline $\mathrm{DBP}, \mathrm{mmHg}$ & $79.37 \pm 10.11^{\mathrm{cd}}$ & $81.33 \pm 9.53^{\mathrm{d}}$ & $83.11 \pm 9.42^{\mathrm{ad}}$ & $85.94 \pm 10.9^{\mathrm{abc}}$ & $P<0.001$ \\
\hline Fasting plasma glucose, $\mathrm{mmol} / \mathrm{L}$ & $4.89(4.57-5.15)$ & $4.95(4.65-5.3)$ & $4.92(4.69-5.34)$ & $4.95(4.69-5.34)$ & 0.008 \\
\hline Insulin, mU/L & $7.78(5.84-10.79)$ & $9.46(6.55-12.69)$ & $9.25(6.35-13.26)$ & $10.86(7.64-14.8)$ & $P<0.001$ \\
\hline HOMA-IR & $1.72(1.24-2.36)$ & $2.06(1.39-2.95)$ & $2.02(1.38-2.99)$ & $2.42(1.67-3.48)$ & $P<0.001$ \\
\hline $\mathrm{RBC}, 10^{12} / \mathrm{L}$ & $4.28(4.15-4.4)$ & $4.57(4.5-4.66)$ & $4.87(4.77-4.97)$ & $5.29(5.11-5.68)$ & $P<0.001$ \\
\hline HGB, g/L & $129.5(124-133)$ & $138(|35-| 4 \mid)$ & $\mid 45(|4|-\mid 49)$ & $155(|49-16|)$ & $P<0.001$ \\
\hline Hct (\%) & $0.4(0.39-0.4 I)$ & $0.43(0.42-0.43)$ & $0.45(0.44-0.46)$ & $0.47(0.46-0.49)$ & $P<0.001$ \\
\hline RDW-SD (fl) & $43.15(41.5-45.33)$ & $43(41.6-44.75)$ & $42.7(40.9-44.53)$ & $42.5(40.5-44.2)$ & 0.001 \\
\hline RDW-CV (\%) & $12.8(12.4-13.7)$ & $13.0(12.5-13.4)$ & $13.1(12.6-13.7)$ & |3.3(|2.9-14.3) & $P<0.001$ \\
\hline WBC, $10^{9} / \mathrm{L}$ & $5.75 \pm 1.39^{\mathrm{bcd}}$ & $6.34 \pm 1.3$ ad & $6.49 \pm 1.32 \mathrm{ad}$ & $6.89 \pm 1.34^{\mathrm{abc}}$ & $P<0.001$ \\
\hline Neutrophil count, $10^{9} / \mathrm{L}$ & $3.01 \pm 1.04^{\mathrm{bcd}}$ & $3.39 \pm 0.97^{\mathrm{ad}}$ & $3.43 \pm 1.01^{\text {ad }}$ & $3.66 \pm 0.97^{\mathrm{abc}}$ & $P<0.001$ \\
\hline Lymphocyte count, $10^{9} / \mathrm{L}$ & $2.12 \pm 0.6^{\mathrm{bcd}}$ & $2.28 \pm 0.6 \mathrm{I}^{\mathrm{ad}}$ & $2.35 \pm 0.62^{\mathrm{a}}$ & $2.46 \pm 0.65^{\mathrm{ab}}$ & $P<0.001$ \\
\hline $\mathrm{IL}-6, \mathrm{pg} / \mathrm{mL}$ & $4.05 \pm 6.95$ & $3.82 \pm 2.17$ & $3.56 \pm 1.9$ & $3.79 \pm 2.24$ & 0.526 \\
\hline $\mathrm{Hs}-\mathrm{CRP}, \mathrm{mg} / \mathrm{L}$ & $2.28 \pm 5.53$ & $2.35 \pm 3.69$ & $2.39 \pm 5.54$ & $2.33 \pm 3.7$ & 0.993 \\
\hline SOD, U/L & $146(\mid 37-157)$ & $149(140-159)$ & $153(144-162.25)$ & $157(\mid 48-169)$ & $P<0.001$ \\
\hline Serum albumin, $g / L$ & $47.17 \pm 2.55^{\mathrm{bcd}}$ & $47.93 \pm 2.55^{\mathrm{ad}}$ & $48.07 \pm 2.4 I^{\text {ad }}$ & $48.76 \pm 2.46^{\mathrm{abc}}$ & $P<0.00 I$ \\
\hline $\mathrm{TG}, \mathrm{mmol} / \mathrm{L}$ & I.I (0.84-I.56) & $1.27(0.92-1.79)$ & $1.28(0.94-1.94)$ & $1.58(1.23-2.25)$ & $P<0.001$ \\
\hline $\mathrm{TC}, \mathrm{mmol} / \mathrm{L}$ & $5.26 \pm 1.05^{\mathrm{bcd}}$ & $5.54 \pm 1.09^{\mathrm{a}}$ & $5.62 \pm 1.06^{\mathrm{a}}$ & $5.54 \pm 1.04^{\mathrm{a}}$ & $P<0.001$ \\
\hline
\end{tabular}

Notes: Data are presented as mean \pm SD or median (25th-75th percentiles) for continuous variables and as percentage (\%) for countable data and categorical variables. One-way analysis of variance between the two groups, Bonferroni: ${ }^{a}$ Indicates comparison with $\mathrm{Q}$ ( $(\mathrm{P}<0.05)$, ${ }^{\mathrm{b}}$ Indicates comparison with $\mathrm{Q} 2(\mathrm{P}<0.05)$, Indicates comparison with Q3 ( $\mathrm{P}<0.05)$, Indicates comparison with Q4 $(\mathrm{P}<0.05)$.

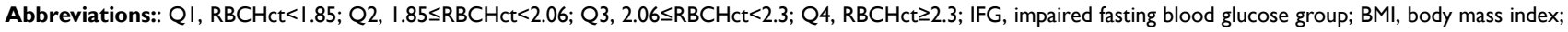
WC, waist circumference; SBP, systolic blood pressure; DBP, diastolic blood pressure; HOMA-IR, insulin resistance homeostasis model assessment; RBC, red blood cell count; HGB, hemoglobin; Hct, hematocrit; RDW-SD, red blood cell distribution width; RDW-CV, coefficient of variation of erythrocyte distribution width; WBC, white blood cell count; IL-6, interleukin 6; hs-CRP, hypersensitive C-reactive protein; SOD, superoxide dismutase; ALB, serum albumin; TG, triglycerides; TG, total cholesterol.

Table 4 The Relationship Between the Interquartile Range of RBCHct Index and the Potential Risk of Impaired Fasting Blood Glucose

\begin{tabular}{|c|c|c|c|c|c|c|}
\hline \multirow{2}{*}{$\begin{array}{l}\text { Interquartile Range } \\
\text { RBCHct }\end{array}$} & \multicolumn{2}{|l|}{ Model I } & \multicolumn{2}{|l|}{ Model 2} & \multicolumn{2}{|l|}{ Model 3} \\
\hline & OR $(95 \% \mathrm{Cl})$ & $\mathbf{p}$ & OR (95\% Cl) & $\mathbf{p}$ & OR $(95 \% \mathrm{Cl})$ & $\mathbf{p}$ \\
\hline $\mathrm{RBCHct}$ & & 0.001 & & 0.002 & & 0.007 \\
\hline QI & I & & 1 & & I & \\
\hline Q2 & $1.854(1.053-3.264)$ & 0.032 & $1.857(1.04 \mid-3.31)$ & 0.036 & $1.756(0.982-3.139)$ & 0.058 \\
\hline Q3 & I.875(I.068-3.292) & 0.029 & $2.018(1.122-3.628)$ & 0.019 & I.889(I.047-3.408) & 0.035 \\
\hline Q4 & $2.925(1.715-4.988)$ & $P<0.001$ & $3.427(1.833-6.407)$ & $P<0.001$ & $3.048(I .6 I 8-5.74 I)$ & 0.001 \\
\hline
\end{tabular}

Notes:: Model I, unadjusted confounding factors. Model 2, adjusted for age, sex, BMI, waist circumference. Model 3, Adjusted for above+ smoking, alcohol use, education (high school or above), physical activity, triglyceride, and total cholesterol.

Abbreviations: RBCHct, red blood cell count multiplied by hematocrit index; OR, odds ratio.

glycated hemoglobin can limit the release of oxygen. ${ }^{18}$ These changes in red blood cells will lead to decreased red blood cell deformability and hypofunction, which in turn leads to secondary red blood cells increase. ${ }^{19}$ (II) Increased hematocrit. Hyperglycemia may cause osmotic diuresis, reducing plasma volume, thereby increasing 


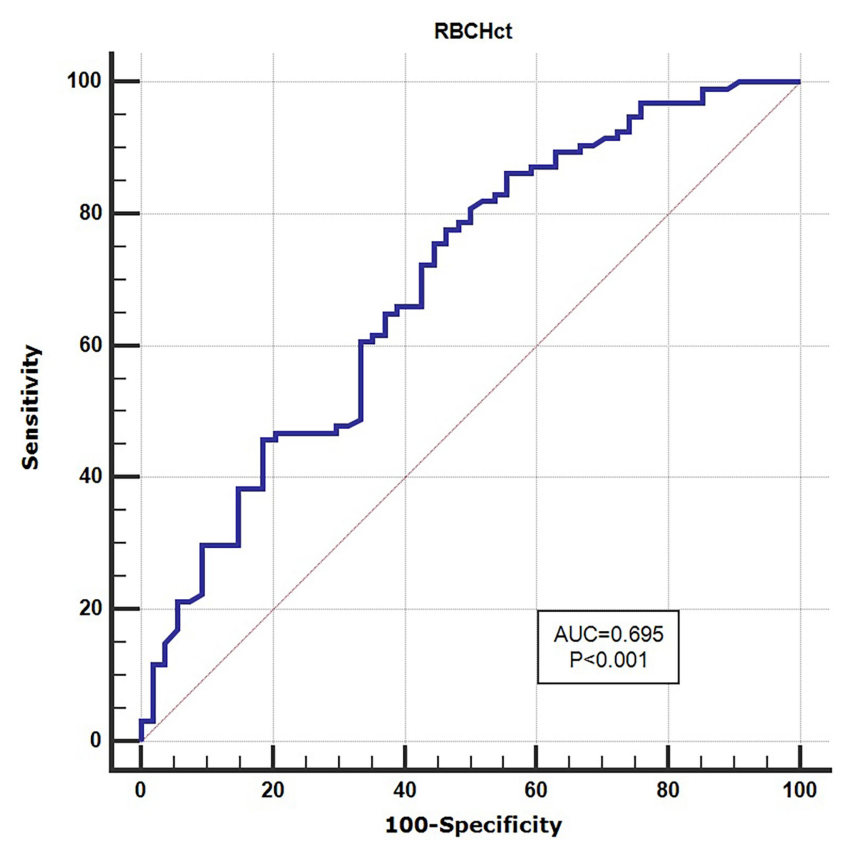

Figure I Characteristics of ROC curve for RBCHct diagnosis of insulin resistance.

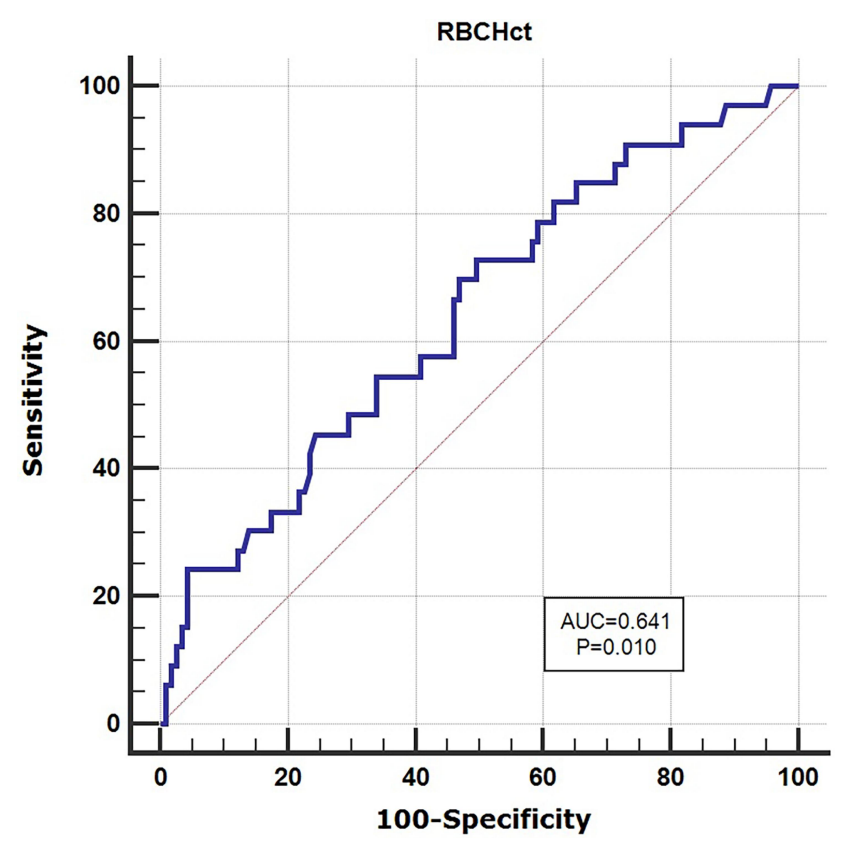

Figure 2 Characteristics of ROC curve of RBCHct diagnosis of chronic low-grade inflammation.

hematocrit, and blood viscosity. ${ }^{20,21}$ Red blood cell function is best when the hematocrit is $0.38 \%$, and then as the hematocrit increases, the ability of red blood cells to carry oxygen gradually decreases. $^{22}$ (III) Red blood cell heterogeneity. Hyperglycaemia can cause red blood cell membrane damage and increase the number of echinocytes and spherocytes. $^{23}$ Previous studies have shown that elevated
RDW-CV indicates increased red blood cell heterogeneity, which is associated with increased risk of diabetes and heart disease. Increased RDW-CV $(\geq 13.1 \%)$ in diabetic patients after percutaneous coronary intervention is associated with increased long-term all-cause mortality. ${ }^{24}$ Our research showed that the RBC and Hct of the IFG group increased compared with the normal group ( $p<0.05)$, and with the increase of the RBCHct index, the RDW-CV showed an increasing trend $(\mathrm{P}<0.001)$. In the binary logistic regression analysis, after adjusting for confounding factors, the highest quartile of RBCHct predicted the risk of IFG to be 3.048 times. Our results indicate that red blood cells may be involved in the occurrence and development of IFG, and the RBCHct index can be used as a potential indicator to predict IFG in patients. When blood glucose increases, the shape and function of RBC change, deformability decreases, the ability to pass capillaries decreases, RBC aggregates, blood viscosity increases, and capillary resistance increases. ${ }^{25-27}$

Previous studies believed that insulin resistance appeared before hyperglycemia and was considered to be the main mechanism of type 2 diabetes. ${ }^{28}$ Insulin resistance can cause changes in red blood cells. The superphysiological dose of insulin combined with the red blood cell insulin receptor will increase the microviscosity of red blood cells, leading to the aggregation of red blood cells and the increase of blood viscosity. ${ }^{29,30}$ Hyperinsulinism activates phosphodiesterase 3 in human red blood cells, impairing the release of adenosine triphosphate (ATP), leading to impaired red blood cell energy supply. ${ }^{31}$ In addition, hyperinsulinemia can promote red blood cells. ${ }^{32}$ Insulin resistance can also be enhanced by changes in red blood cells. Elevated fasting blood glucose will lead to the decrease of insulin receptor kinase activity of red blood cells, fat cells and skeletal muscle, and increase insulin resistance. ${ }^{33}$ The increase of triglycerides and cholesterol in the blood circulation will lead to the destruction of the fatty acid structure of the red blood cell membrane and the increase of membrane cholesterol content, which in turn increases insulin resistance. $^{34,35}$

The correlation between red blood cells and inflammation. The RBC membrane surface molecules can interact with white blood cells, endothelial cells, macrophages, and various blood components, and functionally activate an immune response. ${ }^{36}$ Red blood cells can form microvesicles in response to various physiological and pathological triggers. ${ }^{37}$ Insulin resistance increases the secretion of red blood cell vesicles; and these vesicles which are rich in 
damaged red blood cell components, preferentially combine with circulating white blood cells and change their functions. ${ }^{38}$ In addition, vesicles derived from red blood cells can promote the production of inflammatory cytokines, promote the efficacy of antigen-presenting cells, induce the proliferation of $\mathrm{CD} 4{ }^{+} \mathrm{T}$ and $\mathrm{CD} 8{ }^{+}$ $\mathrm{T}$ lymphocytes, and produce pro-inflammatory effects. ${ }^{39}$ Insulin resistance, red blood cell dysfunction, oxidative stress damage, and increased oxidative stress products are associated with the prediabetes period, and the corresponding superoxide dismutase (SOD) that eliminates oxidative stress products also increases. ${ }^{40,41}$ Similarly, ALB has antioxidant activity and shows an increasing trend in the occurrence and development of diabetes. ${ }^{42-44}$ The imbalance between oxidative and anti-oxidative stress can activate the inflammatory pathway, ${ }^{45}$ and an increase in WBC count indicates inflammation state. ${ }^{46,47}$ The results of our study also showed that the SOD $(\mathrm{P}=0.001)$ and $\mathrm{WBC}$ count $(\mathrm{P}=0.015)$ in the IFG group were higher than those in the normal group, and the RBCHct index and SOD $(r=0.292, \mathrm{P}<0.001)$ and $\mathrm{WBC}(\mathrm{r}=0.305, \mathrm{P}<0.001)$ were highly correlated, and the RBCHct index could predict insulin resistance and chronic inflammation $(\mathrm{P}<0.05)$. This is likely because RBCs are under oxidative stress when blood glucose is elevated, which activates the WBCs in circulation and the innate immune system. ${ }^{36,48}$ This may also mean that during the increase of blood glucose in the body, morphological and functional changes of RBCs, oxidation and anti-oxidation of tissues and organs, and pro-inflammatory and anti-inflammatory reactions have already occurred. ${ }^{48,49}$

This study has certain limitations. First of all, this is a cross-sectional survey with a small sample size and females, older residents account for a larger proportion of our research, which may have the problem of selection bias. Secondly, this study used the form of questionnaire to conduct statistics of past medical history, and patients with diseases that may have an impact on blood system indicators cannot be ruled out. Third, in this study, the sample size of people with IFG was small, the RBCHct index to conduct diagnostic studies on insulin resistance and chronic low-grade inflammation in people with IFG may have low test efficiency. Therefore, further large samples and prospective studies are needed to clarify the significance of the RBCHct index.

\section{Conclusion}

Impaired fasting blood glucose is a high-risk pre-state of diabetes. If the simple and easily neglected blood routine parameter of RBCHct index can be used as an indicator of IFG, insulin resistance and chronic low-grade inflammation in vivo, early intervention measures for high-risk groups may be achieved, thereby reducing the incidence of diabetes and the economic burden of human chronic non-communicable diseases. This study shows that in the IFG population, the number, structure and function of RBCs change, which are manifested by reduced red blood cell deformability, increased aggregation and increased blood viscosity. This may be the result of the interaction with IR, and it may be one of the reasons why the body begins to develop a state of chronic low-grade inflammation. Red blood cells are involved in the development of diabetes and the RBCHct index may be an early indicator of high diabetes risk.

\section{Funding}

This study was supported by Risk factors and prediction model of chronic kidney disease caused by metabolic syndrome: A multicentric prospective cohort study Clinical trial training project of Southern Medical University (LC2016PY047, 2016); Science and Technique Program of Guangzhou (201604020015, 2015); South Wisdom Valley Innovative Research Team Program (CXTD-004, 2014), and The National Natural Science Foundation of China (81873620).

\section{Disclosure}

The authors report no conflicts of interest in this work.

\section{References}

1. Wang L, Gao P, Zhang M, et al. Prevalence and ethnic pattern of diabetes and prediabetes in China in 2013. JAMA. 2017;317 (24):2515-2523. doi:10.1001/jama.2017.7596

2. Banarjee R, Sharma A, Bai S, Deshmukh A, Kulkarni M. Proteomic study of endothelial dysfunction induced by AGEs and its possible role in diabetic cardiovascular complications. $J$ Proteomics. 2018;187:69-79. doi:10.1016/j.jprot.2018.06.009

3. Ceriello A, Motz E. Is oxidative stress the pathogenic mechanism underlying insulin resistance, diabetes, and cardiovascular disease? The common soil hypothesis revisited. Arterioscler Thromb Vasc Biol. 2004;24 (5):816-823. doi:10.1161/01.ATV.0000122852.22604.78

4. Madonna R, De Caterina R. Cellular and molecular mechanisms of vascular injury in diabetes-part I: pathways of vascular disease in diabetes. Vascul Pharmacol. 2011;54(3-6):68-74. doi:10.1016/j. vph.2011.03.005

5. Verma N, Liu M, Ly H, et al. Diabetic microcirculatory disturbances and pathologic erythropoiesis are provoked by deposition of amyloid-forming amylin in red blood cells and capillaries. Kidney Int. 2020;97(1):143-155. doi:10.1016/j.kint.2019.07.028

6. Loyola-Leyva A, Loyola-Rodríguez JP, Terán Figueroa Y, González FJ, Atzori M, Barquera Cervera S. Altered erythrocyte morphology in Mexican adults with prediabetes and type 2 diabetes mellitus evaluated by scanning electron microscope. Microscopy. 2019;68(3):261-270. 
7. Padilla DJ, McDonough P, Behnke BJ, et al. Effects of type II diabetes on capillary hemodynamics in skeletal muscle. Am $J$ Physiol Heart Circ Physiol. 2006;291(5):H2439-H2444. doi:10.1152/ajpheart.00290.2006

8. Cefalu WT, Berg EG, Saraco M, Petersen MP, Uelmen S, Robinson S. Classification and diagnosis of diabetes: standards of medical care in diabetes-2019. Diabetes Care. 2019;42((Suppl 1)): S13-S28. doi:10.2337/dc19-S002

9. Xing X-Y, Yang W-Y, Yang Z-J. The diagnostic significance of homeostasis model assessment of insulin resistance in metabolic syndrome among subjects with different glucose tolerance. Chin J Diabetes. 2004;12(3):182-186.

10. Shah T, Casas JP, Cooper JA, et al. Critical appraisal of CRP measurement for the prediction of coronary heart disease events: new data and systematic review of 31 prospective cohorts. Int $J$ Epidemiol. 2009;38(1):217-231. doi:10.1093/ije/dyn217

11. Pearson TA, Mensah GA, Alexander RW, et al. Markers of inflammation and cardiovascular disease: application to clinical and public health practice: a statement for healthcare professionals from the centers for disease control and prevention and the American Heart Association. Circulation. 2003;107(3):499-511. doi:10.1161/01. CIR.0000052939.59093.45

12. Watała C. In vitro glycation of red blood cell proteins: high levels of glucose lower lipid fluidity of erythrocyte membranes. Exp Pathol. 1988;33(4):233-238. doi:10.1016/S0232-1513(88)80077-X

13. Brownlee M, Vlassara H, Cerami A. Nonenzymatic glycosylation and the pathogenesis of diabetic complications. Ann Intern Med. 1984;101(4):527-537. doi:10.7326/0003-4819-101-4-527

14. Huang M, Que Y, Shen X. Correlation of the plasma levels of soluble RAGE and endogenous secretory RAGE with oxidative stress in pre-diabetic patients. $J$ Diabetes Complications. 2015;29 (3):422-426. doi:10.1016/j.jdiacomp.2014.12.007

15. Viskupicova J, Blaskovic D, Galiniak S, et al. Effect of high glucose concentrations on human erythrocytes in vitro. Redox Biol. 2015;5:381-387.

16. Nigra AD, Monesterolo NE, Rivelli JF, et al. Alterations of hemorheological parameters and tubulin content in erythrocytes from diabetic subjects. Int $J$ Biochem Cell Biol. 2016;74:109-120. doi:10.1016/j.biocel.2016.02.016

17. Gabreanu GR, Angelescu S. Erythrocyte membrane in type 2 diabetes mellitus. Discoveries. 2016;4(2):e60. doi:10.15190/d.2016.7

18. Kar M, Roy A, Bose T, Chakraborti AS. Effect of glycation of hemoglobin on its interaction with trifluoperazine. Protein $J$. 2006;25(3):202-211. doi:10.1007/s10930-006-9003-x

19. Erslev AJ, Caro J. Secondary polycythemia: a boon or a burden? Blood Cells. 1984;10(2-3):177-191.

20. Lowe GD, Lowe JM, Drummond MM, et al. Blood viscosity in young male diabetics with and without retinopathy. Diabetologia. 1980;18(5):359-363.

21. Pries AR, Neuhaus D, Gaehtgens P. Blood viscosity in tube flow: dependence on diameter and hematocrit. Am J Physiol. 1992;263(6 Pt 2):H1770-H1778.

22. Cho YI, Cho DJ. Hemorheology and microvascular disorders. Korean Circ J. 2011;41(6):287-295. doi:10.4070/kcj.2011.41.6.287

23. Revin VV, Klenova NA, Gromova NV, et al. Physical and chemical processes and the morphofunctional characteristics of human erythrocytes in hyperglycaemia. Front Physiol. 2017;8:606. doi:10.3389/fphys.2017.00606

24. Tsuboi S, Miyauchi K, Kasai T, et al. Impact of red blood cell distribution width on long-term mortality in diabetic patients after percutaneous coronary intervention. Circ J. 2013;77(2):456-461. doi:10.1253/circj.CJ-12-0730

25. Lynch EC. Peripheral blood smear. In: Walker HK, Hall WD, Hurst JW, editors. Clinical Methods: The History, Physical, and Laboratory Examinations. Boston: Butterworths Copyright (C) 1990, Butterworth Publishers, a division of Reed Publishing; 1990; Chapter155.
26. Chebbi R. Dynamics of blood flow: modeling of fåhraeus and fåhraeus-lindqvist effects using a shear-induced red blood cell migration model. J Biol Phys. 2018;44(4):591-603. doi:10.1007/s10867018-9508-5

27. Yalcin O, Uyuklu M, Armstrong JK, Meiselman HJ, Baskurt OK. Graded alterations of RBC aggregation influence in vivo blood flow resistance. Am J Physiol Heart Circ Physiol. 2004;287(6):H2644H2650. doi:10.1152/ajpheart.00534.2004

28. Freeman AM, Pennings N. Insulin resistance.[updated $2020 \mathrm{Jul} 10]$. In: StatPearls[Internet]. Treasure Island (FL): StatPearls Publishing; 2020 Jan-

29. Dutta-Roy AK, Ray TK, Sinha AK. Control of erythrocyte membrane microviscosity by insulin. Biochim Biophys Acta. 1985;816 (1):187-190. doi:10.1016/0005-2736(85)90408-0

30. Sloop G, Holsworth RE Jr, Weidman JJ, St Cyr JA. The role of chronic hyperviscosity in vascular disease. Ther Adv Cardiovasc Dis. 2015;9(1):19-25. doi:10.1177/1753944714553226

31. Hanson MS, Stephenson AH, Bowles EA, Sprague RS. Insulin inhibits human erythrocyte cAMP accumulation and ATP release: role of phosphodiesterase 3 and phosphoinositide 3-kinase. Exp Biol Med. 2010;235(2):256-262. doi:10.1258/ebm.2009.009206

32. Barbieri M, Ragno E, Benvenuti E, et al. New aspects of the insulin resistance syndrome: impact on haematological parameters. Diabetologia. 2001;44(10):1232-1237. doi:10.1007/s001250100634

33. Olefsky JM, Nolan JJ. Insulin resistance and non-insulin-dependent diabetes mellitus: cellular and molecular mechanisms. Am J Clin Nutr. 1995;61(4 Suppl):980s-986s. doi:10.1093/ajen/61.4.980S

34. Wang TN, Chang WT, Chiu YW, et al. Relationships between changes in leptin and insulin resistance levels in obese individuals following weight loss. Kaohsiung J Med Sci. 2013;29(8):436-443. doi:10.1016/j.kjms.2012.08.041

35. Bakan E, Yildirim A, Kurtul N, Polat MF, Dursun H, Cayir K. Effects of type 2 diabetes mellitus on plasma fatty acid composition and cholesterol content of erythrocyte and leukocyte membranes. Acta Diabetol. 2006;43(4):109-113. doi:10.1007/s00592-007-0224-4

36. Pretini V, Koenen MH, Kaestner L, et al. Red blood cells: chasing interactions. Front Physiol. 2019;10:945.

37. Leal JKF, Adjobo-Hermans MJW, Bosman G. Red blood cell homeostasis: mechanisms and effects of microvesicle generation in health and disease. Front Physiol. 2018;9:703. doi:10.3389/fphys.2018. 00703

38. Freeman DW, Noren Hooten N, Eitan E, et al. Altered extracellular vesicle concentration, cargo, and function in diabetes. Diabetes. 2018;67(11):2377-2388. doi:10.2337/db17-1308

39. Danesh A, Inglis HC, Jackman RP, et al. Exosomes from red blood cell units bind to monocytes and induce proinflammatory cytokines, boosting T-cell responses in vitro. Blood. 2014;123(5):687-696. doi:10.1182/blood-2013-10-530469

40. Tavares AM, Silva JH, Bensusan CO, et al. Altered superoxide dismutase-1 activity and intercellular adhesion molecule 1 (ICAM-1) levels in patients with type 2 diabetes mellitus. PLoS One. 2019;14(5):e0216256. doi:10.1371/journal.pone.0216256

41. Gunawardena HP, Silva R, Sivakanesan R, Ranasinghe P, Katulanda P. Poor glycaemic control is associated with increased lipid peroxidation and glutathione peroxidase activity in type 2 diabetes patients. Oxid Med Cell Longev. 2019;2019:9471697. doi:10.1155/2019/9471697

42. Halliwell B. Albumin-an important extracellular antioxidant? Biochem Pharmacol. 1988;37(4):569-571. doi:10.1016/0006-2952(88)90126-8

43. Bae JC, Seo SH, Hur KY, et al. Association between serum albumin, insulin resistance, and incident diabetes in nondiabetic subjects. Endocrinol Metab. 2013;28(1):26-32. doi:10.3803/EnM.2013.28.1.26

44. Liu M, Tang J, Zeng J, He Y. Higher serum albumin was related with diabetes incidence and the impact of BMI changes: based on cohort study of 18,384 Chinese male elderly. J Diabetes Complications. 2017;31(12):1663-1668. doi:10.1016/j.jdiacomp.2017.08.015 
45. Spahis S, Borys JM, Levy E. Metabolic syndrome as a multifaceted risk factor for oxidative stress. Antioxid Redox Signal. 2017;26 (9):445-461. doi:10.1089/ars.2016.6756

46. Watts T, Barigou M, Nash GB. Effects of vessel size, cell sedimentation and haematocrit on the adhesion of leukocytes and platelets from flowing blood. Biorheology. 2015;52(5-6):391-404. doi:10.3233/ BIR-15043

47. Zang X, Meng X, Wang Y, et al. Six-year follow-up study on the association between white blood cell count and fasting blood glucose level in Chinese adults: a community-based health examination survey. Diabetes Metab Res Rev. 2019;35(4):e3125. doi:10.1002/ dmrr.3125
48. Rodrigues R, de Medeiros LA, Cunha LM, et al. Correlations of the glycemic variability with oxidative stress and erythrocytes membrane stability in patients with type 1 diabetes under intensive treatment. Diabetes Res Clin Pract. 2018;144:153-160. doi:10.1016/j. diabres.2018.01.031

49. Luc K, Schramm-Luc A, Guzik TJ, Mikolajczyk TP. Oxidative stress and inflammatory markers in prediabetes and diabetes. $J$ Physiol Pharmacol. 2019;70:6.

\section{Publish your work in this journal}

Diabetes, Metabolic Syndrome and Obesity: Targets and Therapy is an international, peer-reviewed open-access journal committed to the rapid publication of the latest laboratory and clinical findings in the fields of diabetes, metabolic syndrome and obesity research. Original research, review, case reports, hypothesis formation, expert opinion and commentaries are all considered for publication. The manuscript management system is completely online and includes a very quick and fair peer-review system, which is all easy to use. Visit http://www.dovepress.com/testimonials.php to read real quotes from published authors. 